\title{
THE ELECTRONIC STRUCTURE OF ILVAITE AND THE PRESSURE AND TEMPERATURE DEPENDENCE OF ITS ${ }^{57} \mathrm{Fe}$ MÖSSBAUER SPECTRUM
}

\author{
B. J. EvanS \\ Department of Chemistry, University of Michigan, Ann Arbor, MI 48109, U.S.A. \\ and \\ G. AMTHAUER \\ Fachbereich Geowissenschaften der Universität, Marburg, Bundesrepublik Deutschland D-3550, Germany
}

(Received 20 June 1979; accepted in revised form 10 January 1980)

\begin{abstract}
Fe}$ Mössbauer measurements have been performed on four, chemically analyzed samples of the mixed-valence mineral ilvaite, $\mathrm{Fe}^{3+} \mathrm{Fe}_{2}{ }^{2+}\left(\mathrm{Si}_{2} \mathrm{O}_{7}\right) \mathrm{O}(\mathrm{OH})$, as polycrystals and oriented-single-crystals between 300 and $475 \mathrm{~K}$ and between 1 and 60,000 bar. At 1 bar and $T<400 \mathrm{~K}$, four absorption features are necessary to account for the spectral envelopes: Three are quadrupole doublets which are assigned to the three crystal/chemical species of iron but not without some ambiguities regarding the appropriate electronic structure. Only the doublet assigned to $\mathrm{Fe}^{2+}$ on $4 c$ (space group Pnma) corresponds to that of an orthodox, high-spin $\mathrm{Fe}^{2+}$ ion. The remaining two doublets are assigned to nominal $\mathrm{Fe}^{2+}$ and $\mathrm{Fe}^{3+}$ on the $8 d$ site but have hyperfine parameters that exhibit anomalous values and temperature dependences, which lead to a coalescence at $\sim 400 \mathrm{~K}$ into a single "Fe ${ }^{3+}$-like" doublet. These phenomena are interpreted as arising from a delocalization primarily of the $d$-electron from the $8 d$ $\mathrm{Fe}^{2+}$ ion into a band-like, itinerant state. For (001) oriented crystals, the influence of pressure is remarkable and features associated with extensive electron delocalization in the high temperature spectra are highly developed even at $30 \mathrm{~kb}$ and $298 \mathrm{~K}$, indicating enhanced electron delocalization as a result of decreased internuclear separations parallel to the infinite ribbons of $8 d$ sites. The spectra of polycrystalline and oriented-(100) and $-(010)$ absorbers change more siowly with pressure and $60 \mathrm{~kb}$ pressure is required to achieve the effects of $30 \mathrm{~kb}$ on a (001) absorber. The fourth feature in the spectrum consists of a broad absorption at $\sim 1.5 \mathrm{~mm} \mathrm{~s}^{-1}$ and is tentatively assigned to an electronic state of minor importance due to electron transfer between localized $\mathrm{Fe}^{2+}$ and $\mathrm{Fe}^{3+}$ centers. The low intensity of this absorption feature, $\sim 10 \%$ of the total, probahly accounts for its neglect by other investigators.
\end{abstract}

\section{INTRODUCTION}

There is a continuing interest in the electronic structure of inorganic and organometallic solids containing at least one chemical element in different oxidation states. Such solids are frequently termed "mixed-valence" solids and often have unusual electronic structures and properties. For the case in which the different (formal) oxidation states occupy the same lattice site, the activation energy for the exchange of an electron between the two valence states is expected to be relatively small or even 0 . Consequently, some of the electrons in such a solid are expected to exist in collective or delocalized electron states appropriate to metallic-like electronic structures. Indeed, extensively delocalized electron states have been observed for a number of mixed-valence inorganic oxides among which $\mathrm{Fe}_{3} \mathrm{O}_{4}[1]$ and $\mathrm{La}_{1-x} \mathrm{~Pb}_{x} \mathrm{MnO}_{3}[2]$ are particularly well-documented cases.

Except for magnetite, i.e. naturally occurring (impure) $\mathrm{Fe}_{3} \mathrm{O}_{4}$, extensive electron delocalization in oxide mineral phases is not well-established even though several transition metal silicate and phosphate minerals have been advanced as possible examples [3,4]. The principal difficulty in the investigation of mineral phases arises from the scarcity of suitable specimens of structures having two oxidation states of the same element on equivalent sites with both oxidation states being essential constituents of the mineral. In the past, most of the investigations of mixed-valence minerals have involved optical spectroscopy; absorption bands in the visible and near IR regions of the spectrum have been attributed to "intervalence charge transfer" and frequently invoked to account for the color and pleochroism of minerals [5]. Definitive application of this technique requires very thin, transparent, and oriented single crystals which are difficult to obtain in the case of strongly absorbing solids. Further complications can arise if other elements substitute on the sites occupied by the mixed oxidation states. Depending on the concentration and crystal/chemical characteristics of the substituting elements, the intrinsic equivalency of the lattice sites may be removed and they become equivalent only in an "averaged" sense. Local fluctuations in, the atomic configurations and geometries occasioned by impurities may lead to an appreciable increase of the activation energy for the process

$$
M_{j}^{n+}+M_{i}^{p+} \rightleftarrows M_{j}^{p+}+M_{i}^{n+}
$$

where $M_{i}^{n+}$ and $M_{j}^{p+}$ refer to the $n$th and $p$ th oxidation states of some metal ion on lattice site $i$ or $j$, respectively. If $i=j$ in eqn (1), i.e. the sites occupied by the two oxidation states are equivalent, then the electron delocalization process would not be thermally activated. However, in addition to an intrinsic inequivalency of 
sites $i$ and $j$, the situation where $i \neq j$ can also arise as a result of impurities ions substituting on neighboring sites and the activation energy for the process in eqn (1) can be appreciable even though the "overall equivalency" of the sites as determined by macroscopic diffraction methods has not been destroyed.

Thus, the interest in the electronic structure of ilvaite, $\mathrm{CaFe}_{2}{ }^{2+} \mathrm{Fe}^{3+}\left(\mathrm{Si}_{2} \mathrm{O}_{7}\right) \mathrm{O}(\mathrm{OH})$, is not surprising since it is reported to have $\mathrm{Fe}^{2+}$ and $\mathrm{Fe}^{3+}$ on equivalent lattice sites and it is not difficult to obtain relatively pure specimens with masses of several tens of grams.

Ilvaite belongs to the sorosilicates with isolated $\mathrm{Si}_{2} \mathrm{O}_{7^{-}}$ groups and hydroxyl-ions in the structure. Structure determinations were performed by Belov and Mokeeva[6], Beran and Bittner[7] and Haga and Takeuchi[8]. The crystal system is orthorhombic with space group Pnma. The Ca atoms are located at the $4 c^{\prime}$ position of Pnma and are surrounded by 60 and 1 hydroxyl ions. Iron occupies two crystallographically distinct positions: (i) the $4 c$ position with the symmetry $m$ and (ii) the $8 d$ position with the symmetry 1 . Both sites are coordinated by six ligands in an approximately octahedral fashion, $4 c$ by $60^{2-}$ ions and $8 d$ by $5 \mathrm{O}^{2-}$ and $1 \mathrm{OH}^{-}$ions.

The structure can be described as a framework formed by $\mathrm{Si}_{2} \mathrm{O}_{7}$-groups and edge-sharing, double chains of $\mathrm{Fe}(8 d) \mathrm{O}_{5}(\mathrm{OH})$, whose 6-fold coordinated interstices are occupied by $\mathrm{Fe}(4 c)$ and whose 7 -fold coordinated interstices are filled by $\mathrm{Ca}\left(4 c^{\prime}\right)$. All naturally occurring ilvaites contain some manganese, but since the Mn content is low, i.e. between 0.5 and $15 \mathrm{wt} \% \mathrm{MnO}$, it has been traditional to treat it as a substitutional impurity. No evidence has been presented, however, to demonstrate that manganese is not essential to the stability of ilvaite. More importantly, however, is the possibility that the $8 d$ sites occupied by the $\mathrm{Fe}^{2+}$ and $\mathrm{Fe}^{3+}$ ions are no longer intrinsically equivalent as a result of the perturbing influence of the $\mathrm{Mn}$ ion.

Assuming for the moment, that $\mathrm{Mn}$ is a non-essential constituent of ilvaite, the ideal chemical formula of ilvaite has a $\mathrm{Fe}^{2+} / \mathrm{Fe}^{3+}$ molar ratio of $2: 1$ and there are several possibilities for the $\mathrm{Fe}^{2+}-\mathrm{Fe}^{3+}$ distribution over the $8 d$ and $4 c$ lattice sites.

(1) $\mathrm{Fe}^{2+}$ and $\mathrm{Fe}^{3+}$ are distributed in a random manner over the $8 d$ and $4 c$ positions. $\mathrm{Fe}^{3+}$.

(2) $8 d$ is occupied completely by $\mathrm{Fe}^{2+}$ and $4 c$ by

(3) $\mathrm{Fe}^{2+}$ and $\mathrm{Fe}^{3+}$ are in $8 d$ and $4 c$ is completely filled by $\mathrm{Fe}^{2+}$.

Despite the concensus of opinion that the cation distribution of ilvaite corresponds to (3), the evidence for this distribution is less than definitive. Even in the most recently reported neutron diffraction investigation of ilvaite [8], the $R$ value was the same for distributions (2) and (3). Excluding the ${ }^{57} \mathrm{Fe}$ Mössbauer investigations of ilvaite from our immediate considerations, the site occupancy has been determined in the past by recourse to interatomic distance arguments. The average internuclear $\mathrm{Fe}-\mathrm{O}$ separations for the $8 d$ and $4 c$ lattice sites are 0.208 and $0.219 \mathrm{~nm}$, respectively, suggesting that the larger $\mathrm{Fe}^{2+}$ ion occupies the $4 c$ and the smaller " $\left(\mathrm{Fe}^{2+}+\right.$
$\left.\mathrm{Fe}^{3+}\right) / 2$ averaged ion" occupies the $8 d$ site. Such internuclear distance arguments are valid only when distinct $\mathrm{Fe}^{2+}$ and $\mathrm{Fe}^{3+}$ ions occupy a lattice site; when there is significant charge delocalization, the actual internuclear distances can be quite different from those calculated using tabulated atomic radii and the agrecment between the experimental and calculated values is not a reliable measure of the site occupancies. For example, in $\mathrm{Fe}_{3} \mathrm{O}_{4}$, the $\mathrm{Fe}-\mathrm{O} B$ site internuclear separation is $0.2065 \mathrm{~nm}$ instead of the $0.208 \mathrm{~nm}$ expected for an "averaged $\left(\mathrm{Fe}^{2+}+\mathrm{Fe}^{3+}\right) / 2$ ion." In fact, the $0.2065 \mathrm{~nm} \mathrm{Fe}(B)-\mathrm{O}$ internuclear separation is closer to that expected for $\left(\mathrm{Fe}^{3+}-\mathrm{O}\right)_{\text {ort }}$, e.g. $0.206 \mathrm{~nm}$. Thus, in considering structureproperty relations for ilvaite, it must be kept in mind that the cation distribution has not been definitively established and that while ${ }^{57} \mathrm{Fe}$ Mössbauer spectroscopy has been particularly useful for resolving questions of site occupancies [9], it has been applied thus far with only qualified success to ilvaite.

The first ${ }^{57} \mathrm{Fe}$ Mössbauer spectroscopic investigation of ilvaite[10], demonstrated the existence of two kinds of $\mathrm{Fe}^{2+}$ ions which would support scheme (3) for the iron distribution. No evidence was reported in this study to indicate electron delocalization between the $\mathrm{Fe}^{2+}$ and $\mathrm{Fe}^{3+}$ ions that would be expected for ions occupying equivalent sites. Evidence for electron delocalization was reported in a subsequent study[11], in which the remarkable changes in the ${ }^{57} \mathrm{Fe}$ Mössbauer spectrum above $250 \mathrm{~K}$, especially the rapid variation in the $a p$ parent quadrupole splittings and isomer shifts of two of the subpatterns, were interpreted in terms of an electron hopping process between $\mathrm{Fe}^{2+}$ and $\mathrm{Fe}^{3+}$. There was a further speculation that ilvaite underwent an "electronic phase transition" between 300 and $350 \mathrm{~K}$ similar to a metal-nonmetal transition[12]. A subsequent and independent investigation[13], confirmed the results of Gerard and Grandjean concerning the temperature dependence of the Mössbauer spectrum but could find no evidence of a crystallographic phase transition by means of X-ray powder diffractometry. It is noteworthy that the only feature of the Mössbauer spectrum cited as evidence for electron delocalization was the change in quadrupole splitting and isomer shifts of two of the subpatterns. Characteristically these earlier results were restricted to high temperature measurements on a limited number of samples. The experimental results were very similar; there was agreement among all three that the spectrum at $300 \mathrm{~K}$ could be interpreted in terms of only three different species of $\mathrm{Fe}$, two $\mathrm{Fe}^{2+}$ and one $\mathrm{Fe}^{3+}$; the evidence cited for electron hopping consisted of rapid evolution of the quadrupole splittings and isomer shifts of two of the patterns; but the suggestion of a metalnonmetal transition by Gerard and Grandjean was rejected by Heilmenn $e t$ al. on the basis of X-ray diffraction measurements: these two groups of investigators advanced very similar interpretations for the temperature dependence of the Mössbauer spectrum.

On the basis of a more recent investigation of a number of analyzed ilvaite specimens the interpretations advanced in these earlier studies of the $298 \mathrm{~K}$ spectrum of ilvaite have been questioned [14]; in addition to the 
unusual temperature dependence of the spectrum, the $298 \mathrm{~K}$ spectrum is interpreted as exhibiting features evincing electron delocalization between $\mathrm{Fe}^{2+}$ and $\mathrm{Fe}^{3+}$ ions. Qualitatively, the conclusions of Amthauer and Evans have been confirmed by a subsequent investigation [15], but the quantitative data reported are limited to one specimen and some important details are lacking, i.e. relative intensity and linewidth data. The goodness-of-fit parameters, $\chi^{2}$ and MISFIT[16], were also unaccountably larger than expected by a factor of two (2) and an order of magnitude, respectively. Since the earlier conference report[14] continuing investigations of the structure and electronic properties of ilvaite have led to results which advance our understanding of its ${ }^{57} \mathrm{Fe}$ Mössbauer spectrum and elucidate the origin of the controversies in the interpretation of the experimental data.

We believe this more definitive set of data has resulted from detailed measurements in a single investigation of several different analyzed specimens as a function of temperature and pressure in polycrystalline and oriented, single-crystal form. Through measurements on different, chemically-analyzed specimens we have investigated the influence of composition on the Mössbauer spectrum of ilvaite; by means of measurements on oriented, single crystals, the details of the relationship of the crystal structure to electron delocalization have been explored and finally, by means of high pressure measurements, we have investigated the infiuence of interatomic distance on electron delocalization. Some of the results of these investigations are presented in this report.

\section{EXPERIMENTAL}

The Mössbauer spectra were recorded with a 1024 channel multi-channel analyzer using a constant acceleration electromechanical drive system with either a sawtooth or a symmetric, triangular velocity wave form. For the latter wave form two mirror symmetric spectra, each collected in 512 channels, were recorded simultaneously and evaluated independently assuming Lorentzian lines; the average of the two resulting parameter sets are reported. The high pressure spectra were all obtained with a symmetric triangular velocity wave form and in some cases the two mirror spectra were combined prior to fitting. For all the experiments a ${ }^{57} \mathrm{Co} / \mathrm{Pd}$ or a ${ }^{57} \mathrm{Co} / \mathrm{Rh}$ source was used. Isomer shifts are reported relative to $\alpha-\mathrm{Fe}$ at room temperature. Polycrystalline absorbers were prepared by grinding under acetone selected crystalline fragments of ilvaite into a fine powder, mixing the powder with a thermosetting plastic powder in a $4: 1$ plastic-to-sample weight ratio, heating the mixture to $100^{\circ} \mathrm{C}$ in circular piston/cylinder die at $\sim 2 \times 10^{3} \mathrm{kPa}$ and cooling rapidly to room temperature. One polycrystalline absorber (see Table 3) was also prepared by placing an acetone slurry on a piece of high purity aluminum foil and permitting the acetone to evaporate to give a circular absorber which was then held firmly between two sheets of the aluminum foil. The absorber thicknesses were $5-10 \mathrm{mg} \mathrm{cm}^{-2}$ of Fe. Details of our standard Mössbauer technique have been reported previously $[19,20]$.
The cell for the high pressure Mössbauer experiments is shown in Fig. 1. In this experimental set-up, the $\gamma$-rays pass through the anvils and absorber parallel to the applied force. The absorption by the boron carbide $\left(\mathrm{B}_{4} \mathrm{C}\right)$ anvils is diminished by a central indentation on the backside. In contrast to diamond anvils also used in high pressure Mössbauer experiments, the relatively large area on the $\mathrm{B}_{4} \mathrm{C}$-anvils $\left(0.5 \mathrm{~cm}^{2}\right)$ allows for the large absorber areas necessary for Mössbauer spectra with good counting statistics. The high pressure cell was developed for use in a clamp device [21], but is now operated directly within the frame of the force-generating press by incorporation of special openings for the source and the detector (see Fig. 1). The calibration of the sample pressure vs applied force was performed by measurements of the electrical resistance of metals with well known, sharp transistions at $25.5 \mathrm{~kb}(\mathrm{Bi}), 39.4 \mathrm{~kb}(\mathrm{Yb})$ and $55.3 \mathrm{~kb}(\mathrm{Ba})$ (Decker $e t$ al.[17]; Cannon[18]). A linear interpolation among these fixed points was adopted. The polycrystalline absorbers were prepared by mixing the powdered sample with epoxy and pressing it into a central hole of $4.0 \mathrm{~mm}$ diameter in a pyrophyllite gasket of $10 \mathrm{~mm}$ outer diameter. Additionally, $30 \mu \mathrm{m}$ thin-sections of ilvaite-sample No. 6-were prepared parallel to $(100),(010)$ and $(001)$, embedded in epoxy and carefully oriented in the central hole of the gasket, so that the propagation direction of the $\gamma$-rays was parallel to the $[100],[010]$ and $[001]$ directions, respectively. The gasket was supported circumferentially by a steel belt and by a malleable mixture of pyrophyllite and epoxy.

The purity of the ilvaite samples was checked by $\mathrm{X}$-ray diffraction. The chemical analyses were performed

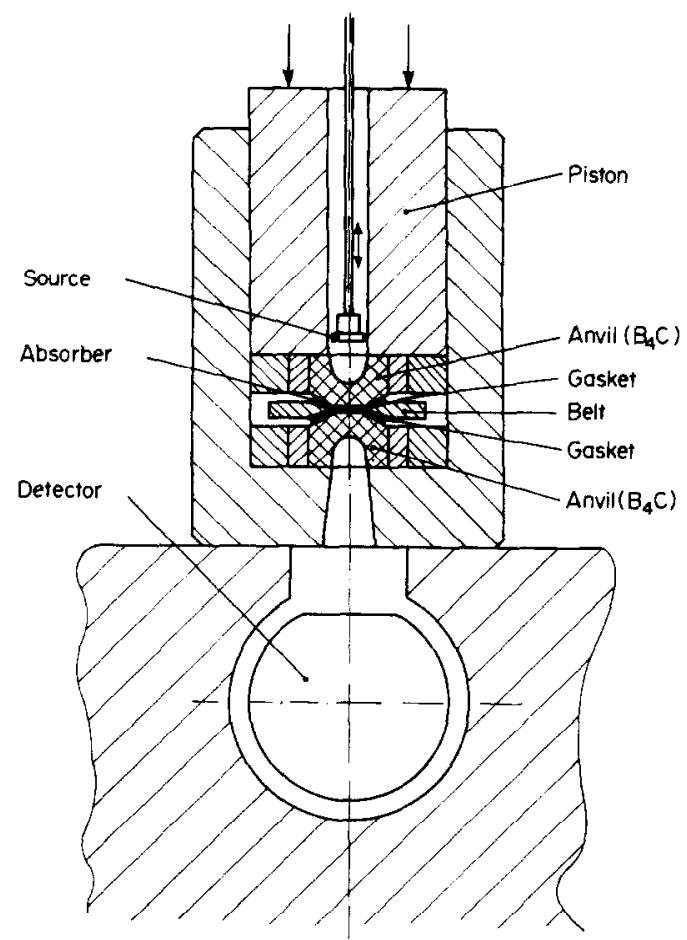

Fig. 1. Cell employed in high pressure ${ }^{57} \mathrm{Fe}$ Mössbauer measurements. 


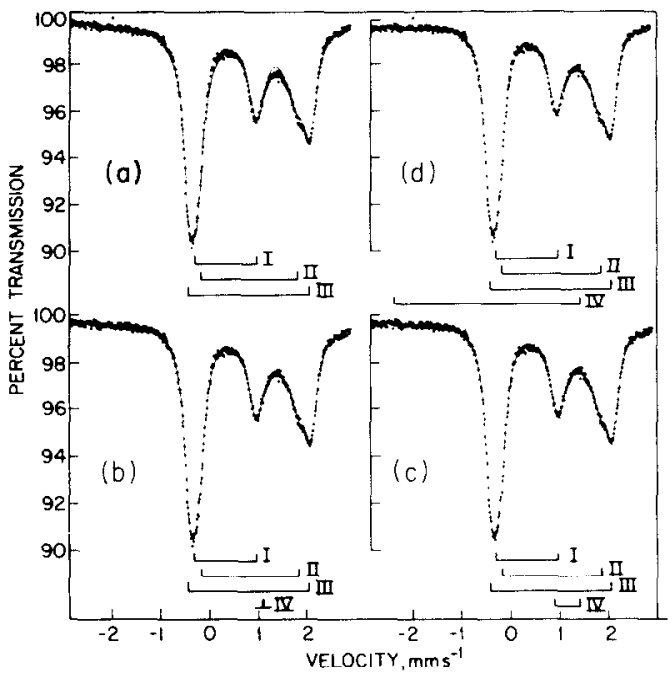

Fig. 2. ${ }^{57} \mathrm{Fe}$ Mössbauer spectrum of sample 6 at $298 \mathrm{~K}$ showing the results $(-)$ of four different models indicated by the bar diagrams. (a) Fit of three quadrupole doublets, exhibiting large deviations between the solid line of the calculated spectrum and the experimental points; (b) fit of three quadrupole doublets and a single-line (subpattern IV); (c) fit of four quadrupole doublets with doublet IV having a small quadrupole splitting; (d) fit of four quadrupole doublets with doublet IV exhibiting a large quadrupole splitting. The fit in 2(d) is the preferred one on quantitative grounds (see Table 3); qualitatively, fits (b) and (c) are, in substance, very similar (see Section 4.1 in text).

by means of an electron microprobe analyzer. For the determination of $\mathrm{Ca}$, a natural apatite crystal was employed as a standard, and for the determination of Fe, $\mathrm{Si}$ and $\mathrm{Mn}$, pure elements were used as standards. The data were corrected and evaluated with the MAGIC IV computer program.

\section{RESULTS}

\subsection{Sample provenance}

The chemical compositions and origins (where known) of the four samples are listed in Table 1 together with an ideal ilvaite composition. In contrast to an ideal ilvaite, all samples contain $\mathrm{Mn}$. The Mn-content varies between $0.35 \mathrm{wt} \%$ (sample 5) and $3.35 \mathrm{wt} \% \mathrm{Mn}$ (sample 7). The increase of $\mathrm{Mn}$ is accompanied by a decrease of the $\mathrm{Fe}$ content. The silicon content is observed to be somewhat high and is believed to result from the use of Si metal as a silicon standard.

\subsection{Mössbauer results}

3.2.1 $298 \mathrm{~K}$ Spectra: Polycrystalline samples. The $298 \mathrm{~K}$ spectrum of sample 6 in Fig. 2 exhibits at least four partially resolved peaks of which three are well into the positive velocity region; and therefore at least three quadrupole doublets are required as a first attempt at fitting the spectrum. The spectrum was therefore initially fitted to three quadrupole doublets under the constraint that

$$
I_{l i} I_{l i}=I_{h i} I_{h i}
$$

where the subscripts $h$ and $l$ indicate the high and low velocity components, respectively, of subpattern $i$. Note that the constraints that $\Gamma_{l i}=\Gamma_{h i}$ and $I_{l i}=I_{h i}$, which are frequently employed in fitting Mössbauer spectra, are included as a special case in eqn (2). The Mössbauer parameters resulting from this fit are given in Table 2 and the statistical parameters in Table 3. The solid line in Fig. 2(a) is the envelope of the fitted spectrum. It is clear from the tables and the figure that the fit according to eqn (2) is inadequate, especially with regard to the large deviation between the experimental points and fitted spectrum near $1.3 \mathrm{~mm} \mathrm{~s}^{-1}$. This three doublet fit is similar to that employed previously by other investigators[1113].

Because of the large deviation between the experimental and fitted spectrum near $1.3 \mathrm{~mm} \mathrm{~s}^{-1}$ (see Fig. 2a) which indicated the presence of an additional absorption, a single line subpattern, in addition to the three quadrupole doublets, was fitted. The envelope of the fitted spectrum is shown in Fig. 2(b) and the hyperfine and statistical parameters of the fit in Tables 2 and 3, respectively. This fit is clearly an improvement over the fit in 2(a). However, the width of the single line subpattern is too broad to be assigned to an intrinsic crystallographic species of iron and the envelope of the fitted spectrum still shows a perceptible deviation from the experimental spectrum near $1.5 \mathrm{~mm} \mathrm{~s}^{-1}$.

The spectrum was therefore fitted as presented in Fig 2(c) to four quadrupole doublets, subject to the constraint of eqn (2) and with one of the doublets being fitted to the broad adsorption between $\sim 1$ and $\sim 1.5$ $\mathrm{mm} \mathrm{s}^{-1}$. This fit results in a quantitative improvement relative to Fig. $2(\mathrm{c})$ in the statistical parameters of the fit and better accounts for the envelope of the spectrum. The relevant parameters are again shown in Tables 2 and 3. Subpattern IV, fitted to the broad absorption is treated as a "quadrupole" doublet only insofar as it accounts for the envelope of the absorption between -1 and $\sim 1.5 \mathrm{~mm} \mathrm{~s}^{-1}$ and serves to define the center of gravity of this absorption feature. The absorption is a very broad one and our use of a doublet pattern is simply a stratagem for fitting the absorption feature more accurately than possible with a single, Lorentzian-shaped line (see Fig. 2(b).

The above fit of four area symmetric quadrupole doublets has the effect of assigning a greater area to the absorptions at high velocity than to those at lower velocities. Since this asymmetric intensity distribution between the high and low velocity regions poses some questions, the spectrum was also fitted to four (4) area symmetric quadrupole doublets under the assumption that the absorption between $\sim 1$ and $\sim 1.5 \mathrm{~mm} \mathrm{~s}^{-1}$ is the high velocity component of a doublet pattern for which the low velocity component is near $-5 x$ $10^{-1} \mathrm{~mm} \mathrm{~s}^{-1}$. The results of fitting four, strongly split doublet patterns to the spectrum are shown in Fig. 2(d), see especially Table 3 . The low velocity component of subpattern IV for this fit occurs at $\sim-2.3 \mathrm{~mm} \mathrm{~s}^{-1}$, well outside of the low velocity absorption region. In addition, the linewidth and isomer shift for this pattern are physically inadmissible. Indeed, the effect of the very broad width of the low velocity component of subpattern 


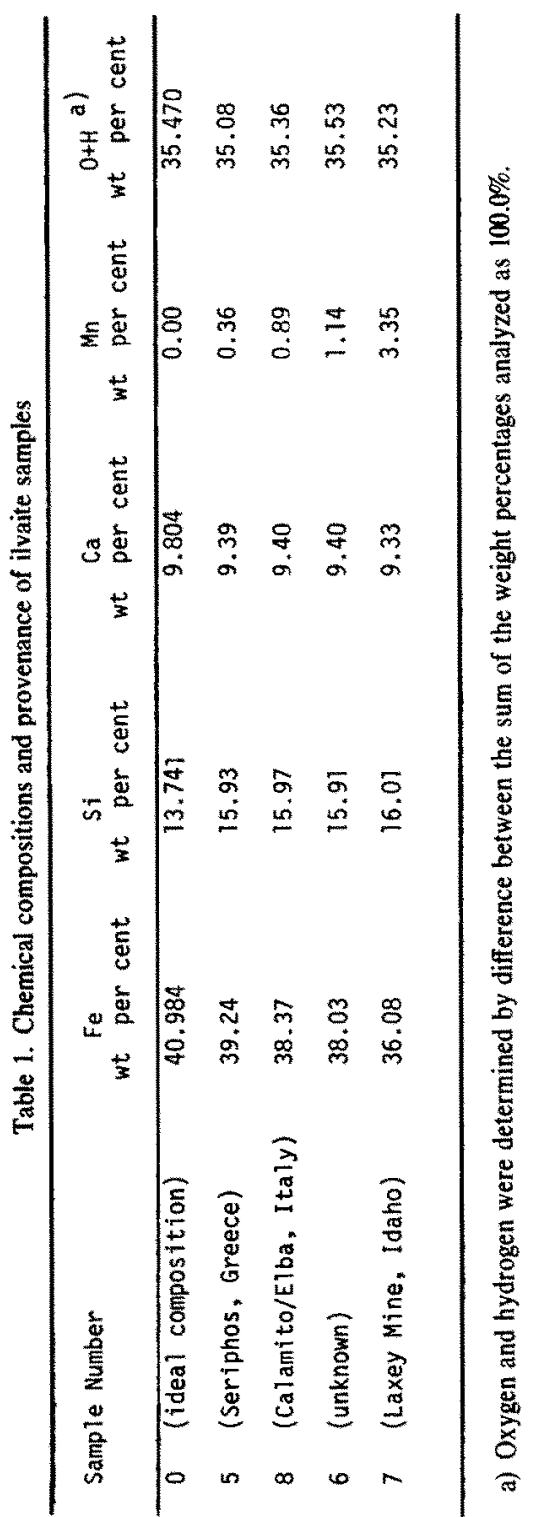



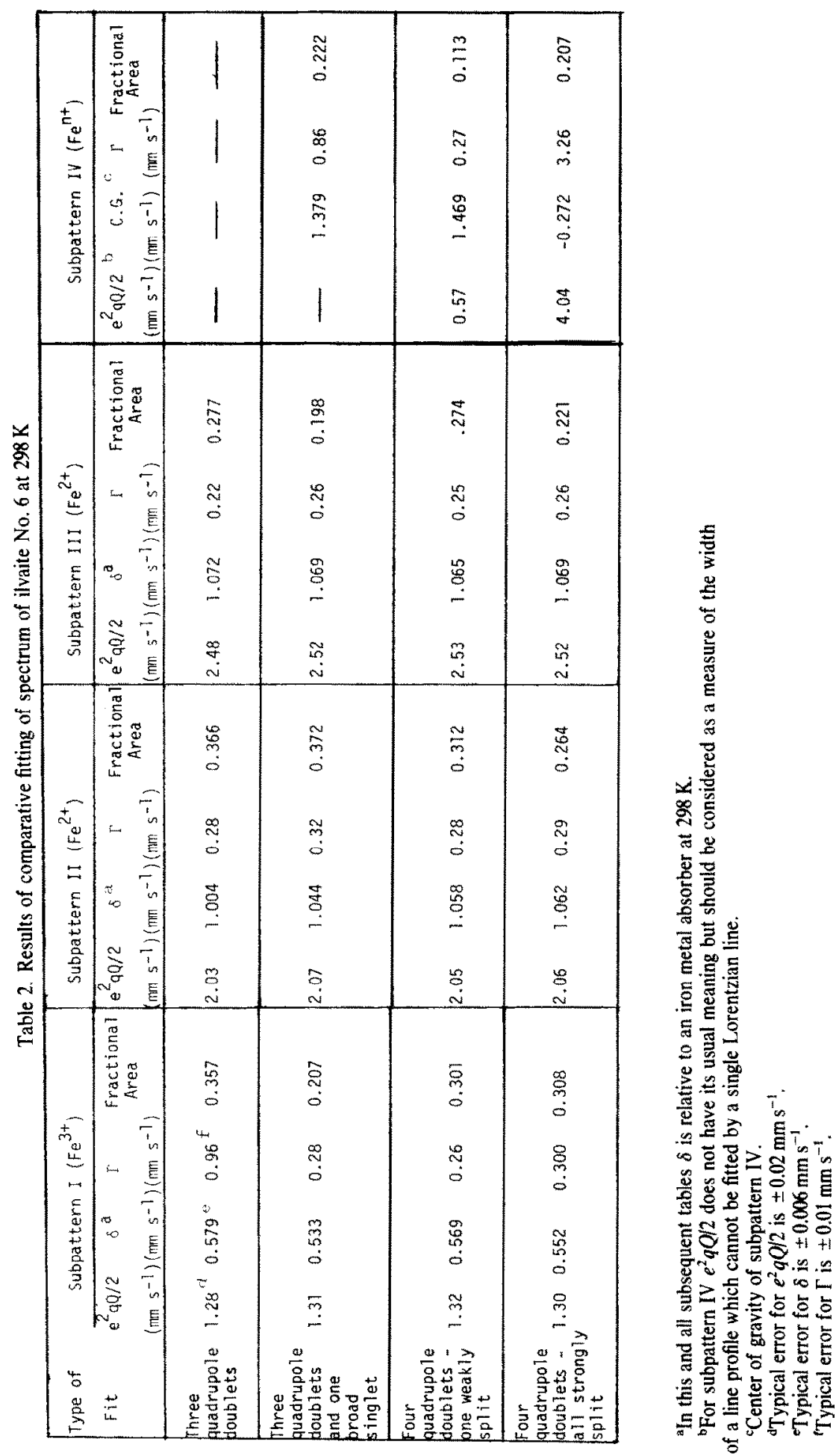
Table 3. Statistical parameters from comparative fitting of two $298 \mathrm{~K}$ spectra of sample 6

\begin{tabular}{|c|c|c|c|c|}
\hline Spectrum ${ }^{a}$ & $\mathrm{Fit}^{\mathrm{b}}$ & Counts * $10^{6}$ & $x^{2 c}$ & MISFIT \\
\hline 1 & $\begin{array}{l}3 \text { doublets } \\
3 \text { doublets, } 1 \text { line } \\
4 \text { doublets }{ }^{2} \\
4 \text { doublets }\end{array}$ & $\begin{array}{l}1.379 \\
1.379 \\
1.379 \\
1.379\end{array}$ & $\begin{array}{r}9442 \\
1079 \\
952 \\
859\end{array}$ & $\begin{array}{l}0.496 \\
0.032 \\
0.022 \\
0.021\end{array}$ \\
\hline 2 & $\begin{array}{l}3 \text { doublets } \\
3 \text { doublets, I line } \\
4 \text { doublets } c \\
4 \text { doublets }^{d}\end{array}$ & $\begin{array}{l}1.144 \\
1.144 \\
1.144 \\
1.144\end{array}$ & $\begin{array}{r}1525 \\
621 \\
593 \\
576\end{array}$ & $\begin{array}{l}0.189 \\
0.026 \\
0.021 \\
0.018\end{array}$ \\
\hline
\end{tabular}

(a) Spectra 1 and 2 represent two independent data sets collected on different spectrometer systems.

(b) The areas of the high and low velocity components of each doublet were constrained to be equal

(c) For 500 data points.

(d) 4th doublet, centered near $-1.5 \mathrm{~mm} \mathrm{~s}^{-1}$ and with a small quadrupole splitting of $-5 \times 10^{-1} \mathrm{~mm} \mathrm{~s}^{-1}$ (see Table 2).

(e) 4th doublet, centered near $\sim-0.3 \mathrm{~mm} \mathrm{~s}^{-1}$ and with a large quadrupole splitting of $\sim 4 \mathrm{~mm} \mathrm{~s}^{-1}$ (see Table 2).

IV is to reduce the amplitude of the intensity to the level of the statistical fluctuations in counts: there is effectively no low velocity component. The high velocity component has parameters virtually identical to those resulting from Fig. 2(b), i.e. the single line fit to the absorption between $\sim 1$ and $1.5 \mathrm{~mm} \mathrm{~s}^{-1}$.

Fits involving as many as eight independently variable lines also demonstrated that the absorption area in the high velocity region exceeded that in the low velocity region, confirming the results of our constrained fits. The spectra of ilvaites have therefore been fitted as in Fig. 2(c) to four doublets, three with large splittings that are assigned to, more or less, well-defined crystal/chemical states of iron and one with a small splitting for which the hyperfine parameters do not have their usual meanings. For subpattern IV only the center of gravity and the total breadth of the absorption profile have crystal chemical and crystal physical significances.

The $298 \mathrm{~K}$ Mössbauer spectrum of sample 6 was obtained on an entirely different spectrometer from the one used to obtain the results presented above and the procedure for evaluating the spectra was repeated. The Mössbauer parameters were very similar and only the statistical parameters of the fit are shown in Table 3. The trends are the same as in those observed for the first set of results and the reproducibility of our evaluation procedure is confirmed.

The $298 \mathrm{~K}$ Mössbauer spectra of samples 5-8 are shown in Fig. 3 and the parameters for the four subpattern fits are listed in Table 4. Quadrupole splittings and isomer shifts reveal some variations slightly exceeding the experimental error which is less than $\pm 0.02 \mathrm{~mm} \mathrm{~s}^{-1}$ for the parameters listed. The areas of the four subpatterns show distinct differences, especially for subpattern IV, which varies between $7.85 \%$ (sample 5) and $11.29 \%$ (sample 6); but do not correlate with $\mathrm{Mn}$ content.

3.2.2 High temperature spectra: Polycrystalline sample. Spectra for sample 5 were obtained at elevated temperatures between 310 and $475 \mathrm{~K}$ and selected spec-

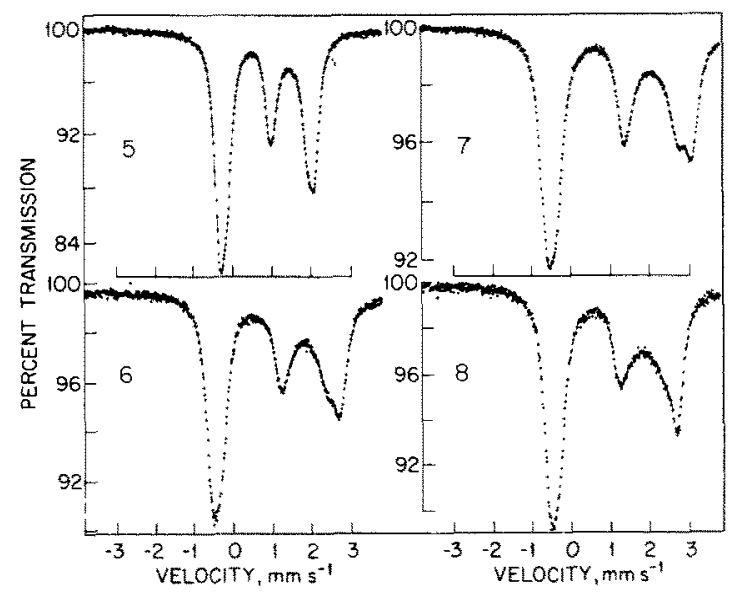

Fig. 3. ${ }^{57} \mathrm{Fe}$ Mössbauer spectra at $298 \mathrm{~K}$ of the four samples investigated in this study. The solid lines are the envelopes of the fits corresponding to the fitting scheme in Fig. 2(c).

tra are presented in Figs. 4 and 5. Between 298 and $330 \mathrm{~K}$ four resolved lines are observed, in accordance with the results presented above for the $298 \mathrm{~K}$ spectra; above $330 \mathrm{~K}$, however, only three lines are clearly resolved. Between 298 and $330 \mathrm{~K}$ the fitting model applied to spectrum $2(c)$ results in statistically acceptable fits. The Mössbaner parameters of the four subpatterns are listed in Table 5 and are observed to exhibit well-defined variations with temperature. The variations are most pronounced for subpattern II and are characterized by a rapid decrease in the magnitude of the quadrupole splitting and isomer shift and a less dramatic decrease in integrated intensity (area). Subpattern I does not exhibit dramatic changes in the magnitudes of the hyperfine parameters but the direction of the changes is anomalous. Instead of decreasing as expected, the integrated intensity, isomer shift and quadrupole interaction increase with increasing temperature. The parameters of subpattern III exhibit a behavior usually observed for a crystal chemically well-defined $\mathrm{Fe}^{2+}$ ion. 

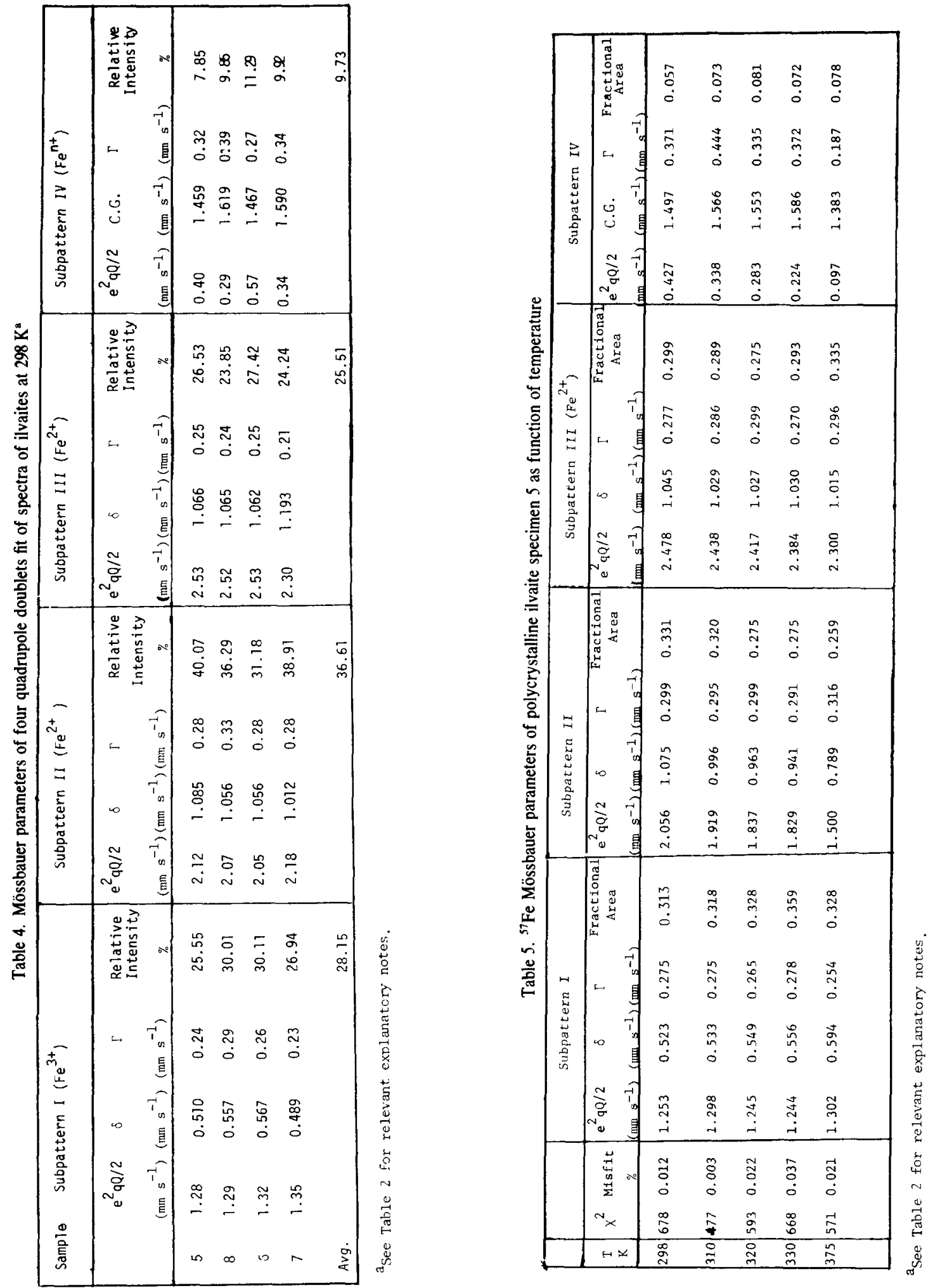


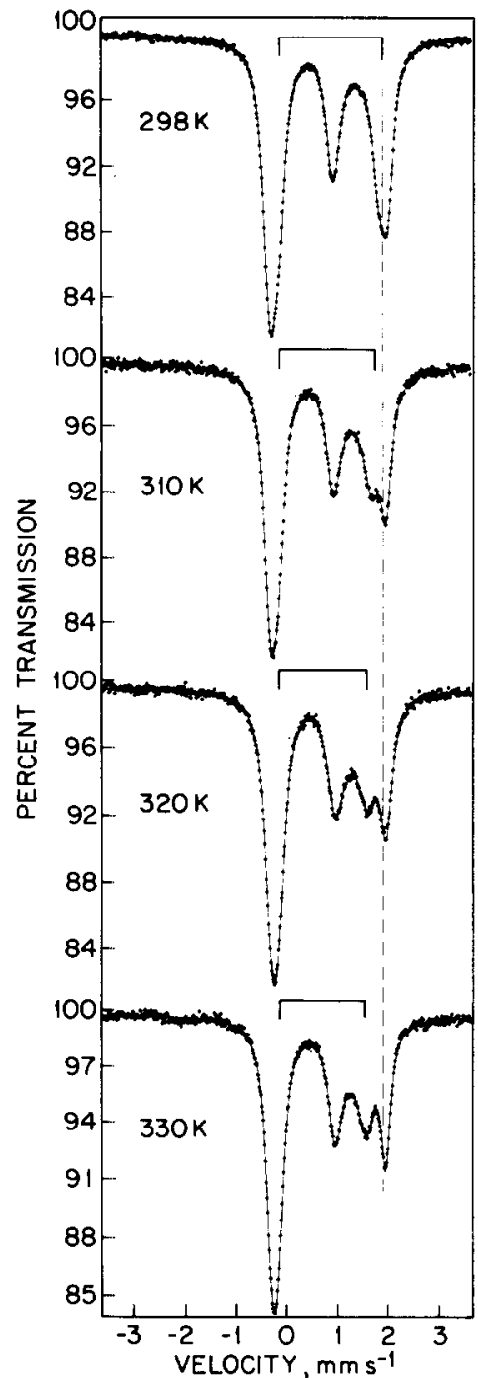

Fig. 4. ${ }^{57} \mathrm{Fe}$ Mössbauer spectra of sample 5 at a number of different temperatures. (-) represent four quadrupole doublet fits as described in Figs. 2 and 3.

These results are in agreement with those of some of the previously reported investigations [11, 13].

The four subpattern fits employed above cannot be uniquely applied to the specra obtained at 425 and $475 \mathrm{~K}$, shown in Fig. 5 . At these temperatures, the high velocity components of subpatterns I and II, as well as both components of IV, are so close together that a fit of four lines to this single adsorption feature is unreliable. Consequently, the spectra have been fitted to two subpat-

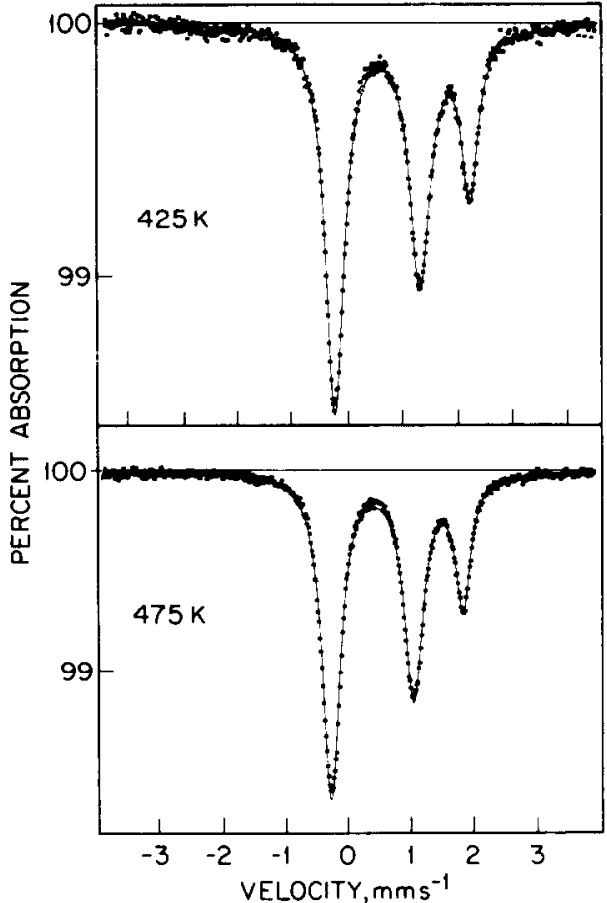

Fig. 5. ${ }^{57} \mathrm{Fe}$ Mössbauer spectra of sample 5 at 425 and $475 \mathrm{~K}$. The solid line is the envelope of a two-quadrupole-doublet fit, subject to the constraint that both components of each doublet have the same integrated intensity.

terns for which the solid line through the data points in Fig. 5 corresponds to the envelope of the fitted spectrum. The parameters resulting from the fitting are shown in Table 6. Subpattern III in Table 6 is identified with subpattern III in Tables 4 and 5. Subpattern V of Table 6 has no counterpart in the lower temperature spectra. The ratio of the area of subpattern $V$ to that of subpattern III is close to the value of 2 which one might expect if subpattern $V$ results from a coalescence of subpatterns I-III. The temperature dependence of the hyperfine parameters of subpatterns III and V exhibit none of the anomalies observed at lower temperatures. Our high temperature results are in good accord with those of Gerard and Grandjean, and Heilmann et al,[11-13].

3.2.3 High pressure spectra: Polycrystalline sample. Typical ${ }^{57} \mathrm{Fe}$ Mössbauer spectra at high pressures of a polycrystalline specimen taken from the same parent crystal as sample 6 are shown in Fig. 6. It has been possible to fit these data with a model similar to that employed for the $298 \mathrm{~K}$, ambient pressure spectra. The results of the fitting are shown in Table 7.

Table 6. ${ }^{57} \mathrm{Fe}$ Mössbauer parameters of ilvaite specimen 5 at $425 \mathrm{~K}$ and $475 \mathrm{~K}$

\begin{tabular}{|c|c|c|c|c|c|c|c|c|c|}
\hline \multirow[b]{2}{*}{$\mathrm{T}$} & \multirow[b]{2}{*}{$x^{2}$} & \multicolumn{4}{|c|}{ Subpattern III $\mathrm{Fe}^{2+}$} & \multicolumn{4}{|c|}{ Subpattern $\mathrm{V}$} \\
\hline & & $\begin{array}{l}\mathrm{e}^{2} \mathrm{qQ} / 2 \\
\left(\mathrm{~mm} \mathrm{~s}^{-1}\right)\end{array}$ & $\begin{array}{c}\delta \\
\left(\mathrm{mm} \mathrm{s} \mathrm{s}^{-1}\right)\end{array}$ & $\begin{array}{c}\Gamma \\
\left(\operatorname{mm} \mathbf{s}^{-1}\right)\end{array}$ & $\begin{array}{l}\text { Fractiona1 } \\
\text { Area }\end{array}$ & $\begin{array}{r}\mathrm{e}^{2} \mathrm{qQ} / 2 \\
\left(\mathrm{~mm} \mathrm{~s}^{-1}\right)\end{array}$ & $\begin{array}{c}\delta \\
\left(\mathrm{mm} \mathrm{s}^{-1}\right)\end{array}$ & $\begin{array}{c}\Gamma \\
\left(\min s^{-1}\right)\end{array}$ & $\begin{array}{l}\text { Fractional } \\
\text { Area }\end{array}$ \\
\hline 425 & 629 & 2.158 & 1.002 & 0.314 & 0.326 & 1.363 & 0.670 & 0.398 & 0.674 \\
\hline 475 & 1100 & 2.094 & 0.972 & 0.301 & 0.328 & 1.304 & 0.577 & 0.361 & 0.672 \\
\hline
\end{tabular}




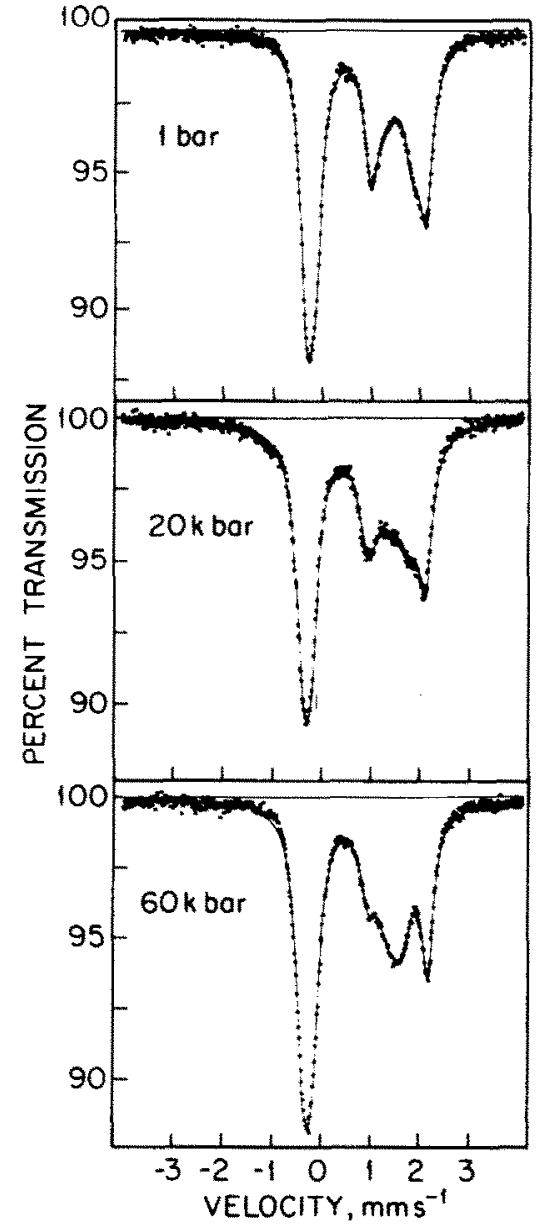

Fig. 6. ${ }^{57} \mathrm{Fe}$ Mössbauer spectra of a polycrystalline absorber of sample 6 at $298 \mathrm{~K}$ and at (a) I bar; (b) $30 \mathrm{kbar}$ and (c) $60 \mathrm{kbar}$ pressure.

A number of aspects are noteworthy. First, the splitting of pattern II decreases appreciably with increasing pressure; the decrease in the splitting of subpattern IV should be considered as a decrease in the linewidth. Secondly, there are significant variations in the intensity of subpatterns I and II, which are obvious in Fig. 6.

Relative to the temperature dependence of the spectra (see Section 3.2.2), the most significant result of the high pressure measurements on the polycrystalline sample is the appreciable decrease in the quadrupole splitting and isomer shift of subpattern II. All subpatterns exhibit a decreasing isomer shift with increasing pressure, as expected, but the decrease in the isomer shift of subpattern Il is at least a factor of three larger than those for the other subpatterns. The decrease in the quadrupole splitting of subpattern II is also rather rapid. In these aspects high pressure and high temperature below $320 \mathrm{~K}$ have similar effects on the spectrum.

3.2.4 High pressure spectra: Single crystal samples. Spectra of $(100),(010)$ and $(001)$ single crystal platelets of ilvaite at several pressures are shown in Figs. 7-9. These single crystal platelets were obtained from the same parent crystal (No. 6) as the polycrystalline sample for which analogous results are reported in Sec.

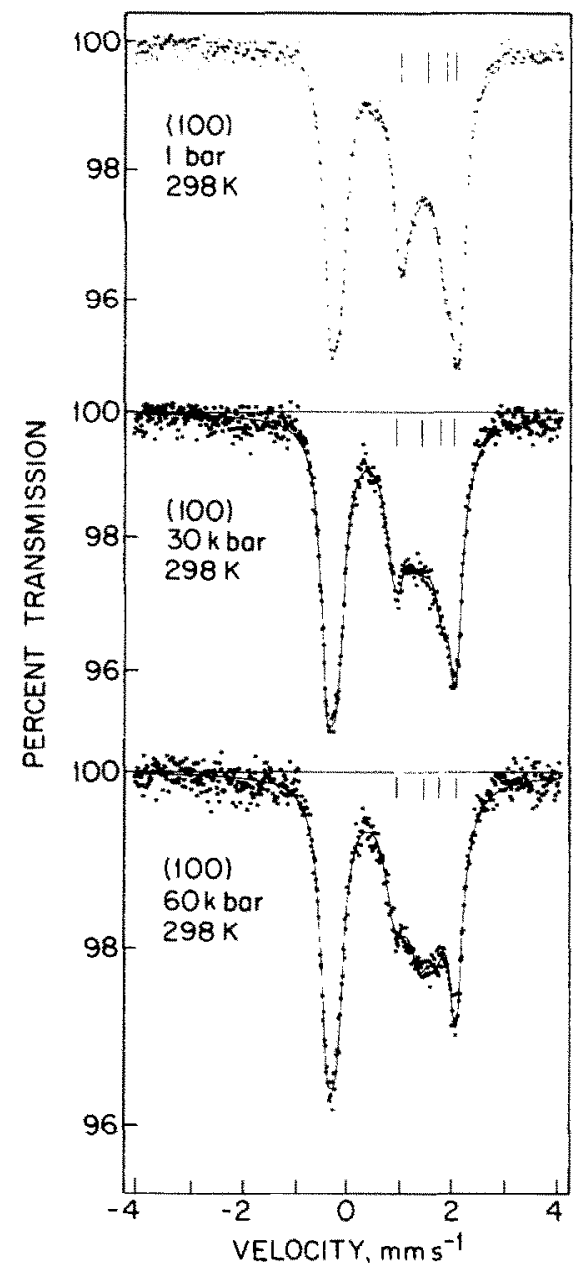

Fig. 7. ${ }^{57} \mathrm{Fe}$ Mössbauer spectra of a (100) single crystal platelet of sample 6 at $298 \mathrm{~K}$ and three different pressures. The greater intensity of the absorption near $2 \mathrm{~mm} \mathrm{~s}^{-1}$ compared to that in Fig. 2 is noteworthy. The spectra were fitted as in Fig. 2(b) and the vertical lines at the top of each spectrum indicate the positions of the high velocity components of subpatterns I, IV, II and III, respectively, with increasing velocity.

tion 3.2.3. It is not possible, however, to fit these spectra in precisely the same manner as the high pressure spectra of the polycrystalline sample insofar as the relative intensities are concerned. The relative intensities of the components of a quadrupole doublet pattern or subpattern in a Mössbauer spectrum is orientation dependent according to the relationships given below:

$I \propto 3 / 2\left(1+\cos ^{2} \theta\right)$ for the $\pm 3 / 2 \rightarrow \pm 1 / 2$ transition,

and

$I \propto 1+3 / 2 \sin ^{2} \theta \quad$ for the $\pm 1 / 2 \rightarrow \pm 1 / 2$ transition,

where $I$ is the relative intensity, $\underline{\theta}$ is the angle between the $\gamma$-ray propagation direction and the principal axis of the electric field gradient tensor (EFG). Other orientation dependent variations in intensity can also result from an anisotropic recoilless fraction. Therefore, for single crystals, the components of a given quadrupole doublet will not in general have equal intensities; if $\theta=0$, the relative intensities will exhibit their maximum difference 


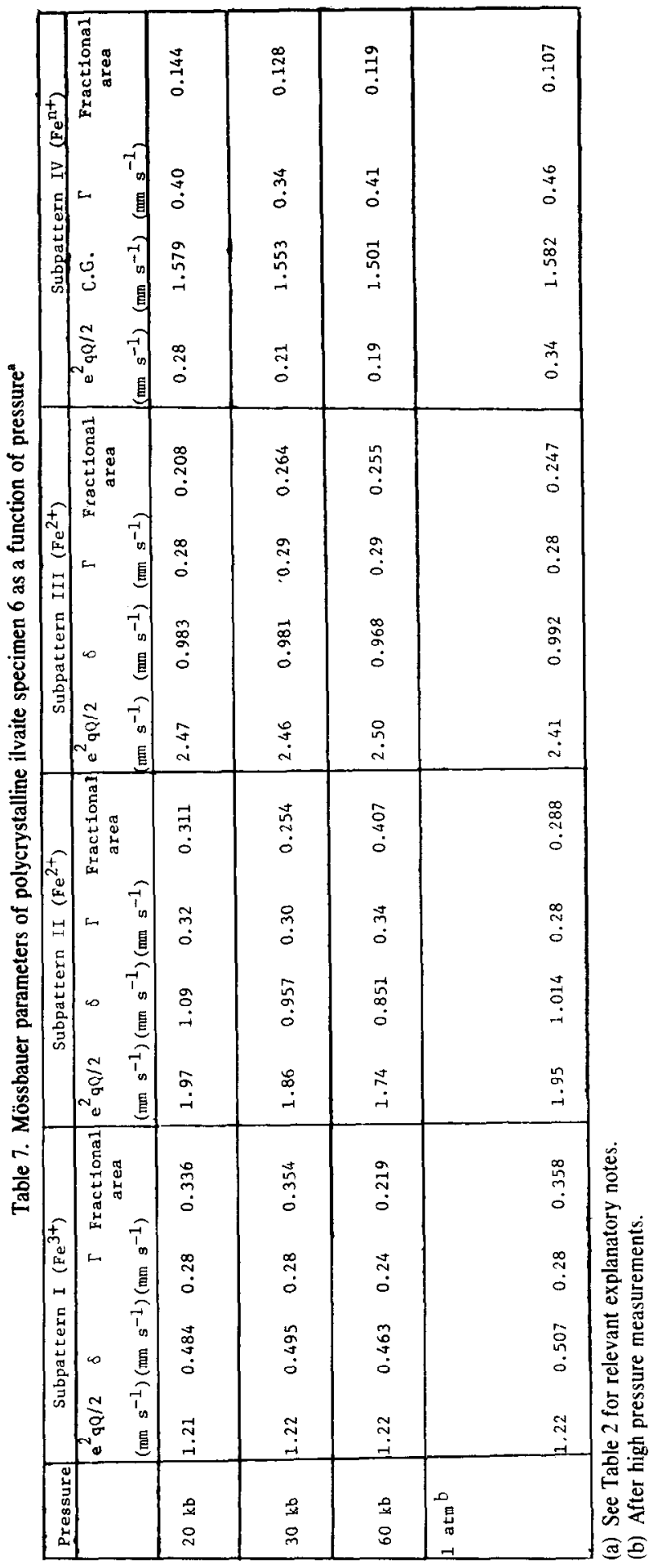




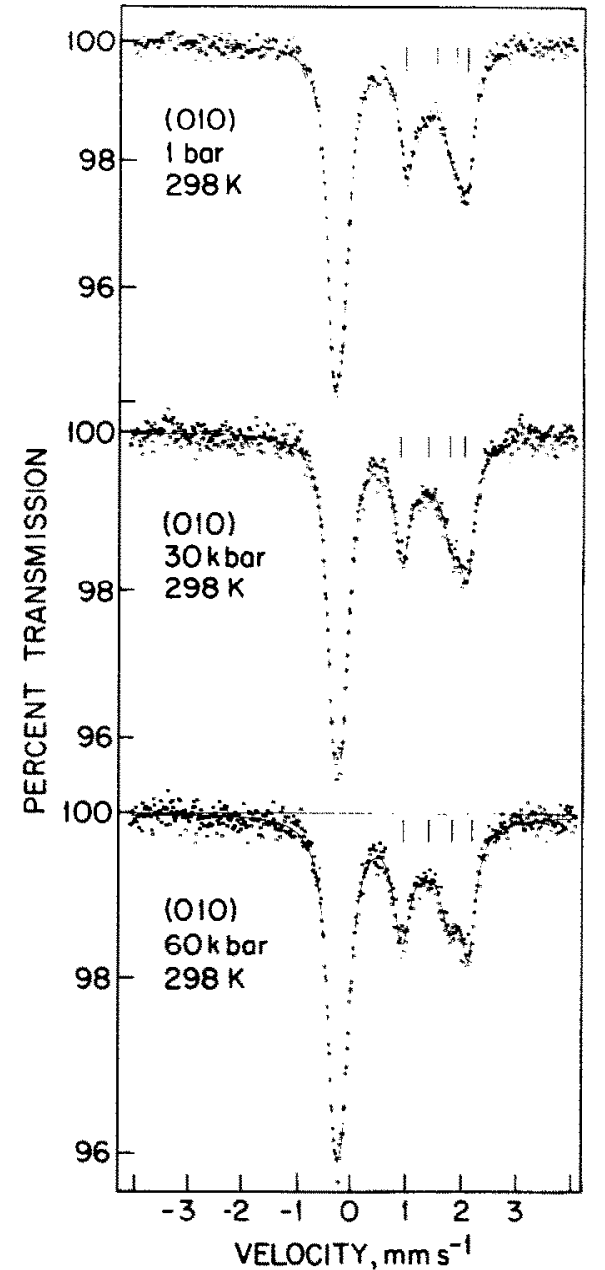

Fig. 8. ${ }^{57} \mathrm{Fe}$ Mössbauer spectra of a (010) single crystal platelet of sample 6 at $298 \mathrm{~K}$ and at $1 \mathrm{bar}, 30 \mathrm{kbar}$ and $60 \mathrm{kbar}$ pressure. The similarity of the spectra to those of a polycrystalline sample at ambient pressurc and the relatively small changes with increasing pressure are cogent aspects of the spectra.

of $3: 1$; at $\theta=90^{\circ}$, the relative intensities are $3: 5$. Whether the high or low velocity line is the more intense depends upon the sign of the EFG. These effects are clearly evident upon comparing the spectra in Figs. 7-9 with those in Fig. 6. For example, on the one hand the (010) and (001) spectra at ambient pressure are not noticeably different from that of the polycrystalline sample. On the other hand, the intensity ratio of the high to low velocity component of subpattern III, at least, is considerably larger for the (100) platelets than for any of the other samples. Incidentally, this result suggests that [100] is close to one of the symmetry axes of the EFG for subpattern III.

Consequently, for a structure such as ilvaite in which both the orientations and signs of the EFG at different sites can be different, the area ratio constraints employed in the analysis of the spectra of polycrystalline samples are not applicable. It is still possible to fit the data but for such strongly overlapping lines, the fits can be extremely degenerate. The lines drawn through the data points in Figs. 7-9 are the results of fitting the spectra with the same number of patterns as employed for the ambient

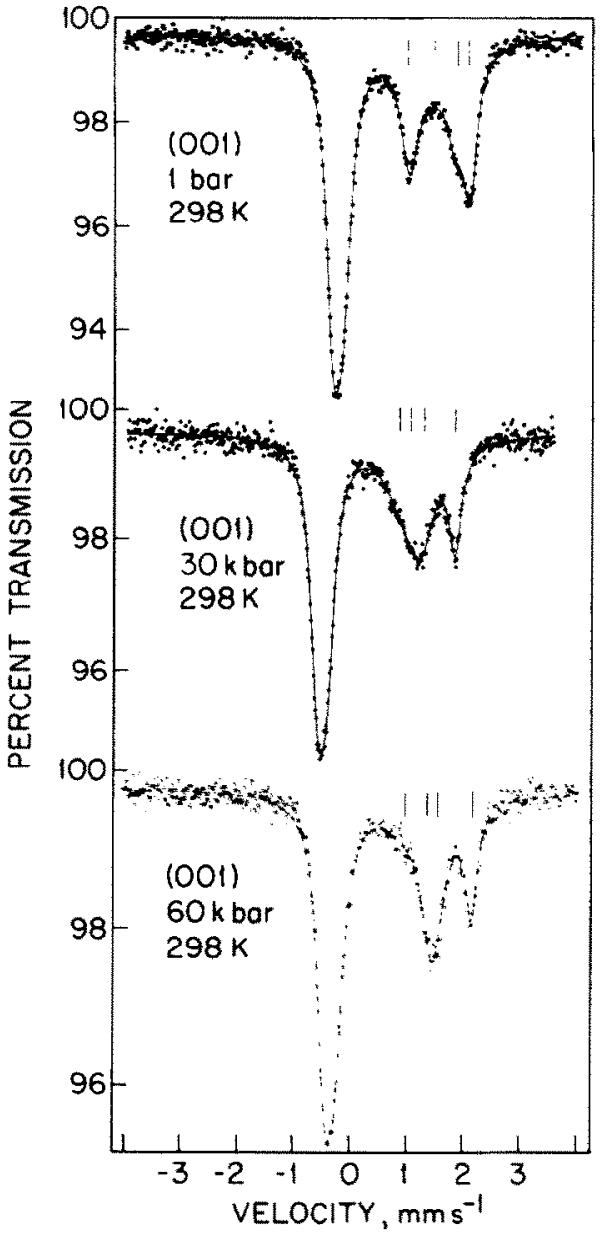

Fig. 9. ${ }^{57} \mathrm{Fe}$ Mössbauer spectra of a (001) single crystal platelet of sample 6 at $298 \mathrm{~K}$ and at $1 \mathrm{bar}, 30 \mathrm{kbar}$ and $60 \mathrm{kbar}$ pressure. The vertical lines at the top of each spectrum have the same meaning as in Fig. 7. The much stronger pressure dependence of the hyperfine interaction parameters of subpattern II compared to that exhibited in Figs. 7, 8 is noteworthy.

pressure spectra but with a relaxation of the constraint that the areas of the high and low velocity components of a given subpattern be equal. For these spectra, a single line fit to subpattern IV resulted in the same goodness-of-fit parameters as a doublet fit. Even though the intensities obtained in the singlet and doublet fits to subpattern IV were rather different, their centers-ofgravity were quite similar. A detailed listing of the parameters resulting from the fits is not desirable since the values of some are very model dependent and only the line positions of the high velocity components are considered to be reliable. The positions of the high velocity components of the subpatterns are indicated by the vertical bars in the figures and the variations in the spectra with pressure can be easily followed by means of the shifts in position of these bars. Subpattern IV is indicated by a single bar giving the position of its center of gravity.

The significant results, which are independent of the fitting model, are as follows: (1): For the (100) and (010) platelets, the primary effect of high pressures is to shift the high velocity component of subpattern II to lower 


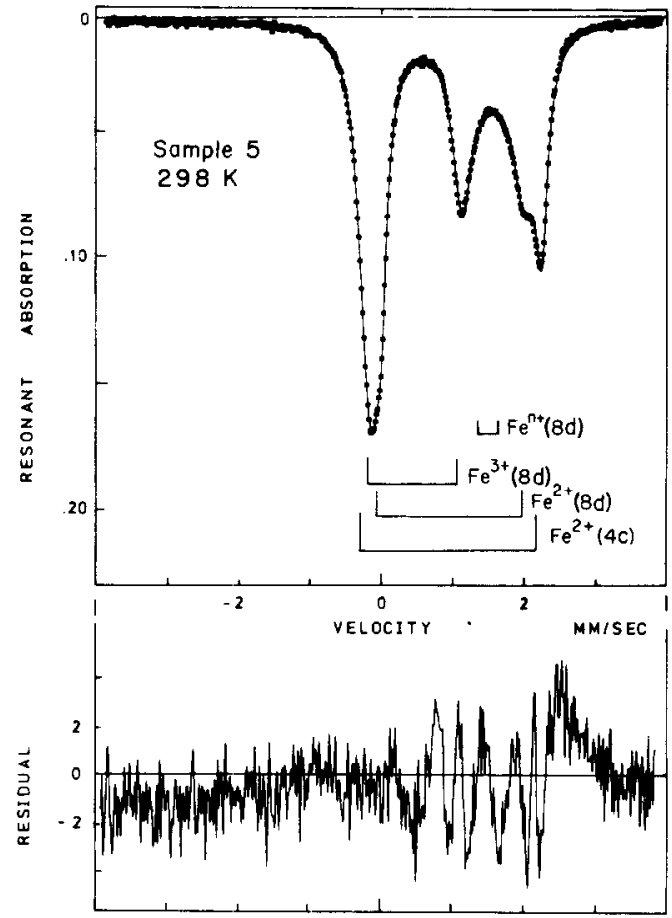

Fig. 10. ${ }^{57} \mathrm{Fe}$ Mössbauer spectrum of sample 5 at $298 \mathrm{~K}$, showing the assignment of the different subpatterns to the different crystallographic and electronic species of $\mathrm{Fe}$ ions.

velocities, i.e. to decrease the apparent quadrupole splitting of this subpattern. (2): For the (001) platelet, the effect of pressure is more dramatic. Qualitatively, there is a greater redistribution of intensity and line positions at $30 \mathrm{~kb}$ for the $(001)$ platelet than at $55 \mathrm{~kb}$ for the $(100)$ and $(010)$ platelets, and polycrystalline sample. As in the case of the other single crystals, the shifting of the high velocity component of subpattern II to more negative velocities is clearly one of the major effects of increasing pressure. The fitting results indicate an increase in the intensity of subpattern IV but measurements at lower pressures are needed to verify this. For the (001) platelet, the positions of the lines do not change appreciably between 30 and $60 \mathrm{~kb}$; the principal change is a decrease in the linewidths.

\section{DISCUSSION}

4.1 Room temperature spectra: Polycrystalline sample

The two doublets (subpatterns II, III) with large quadrupole splittings and the high isomer shifts are assigned to octahedral $\mathrm{Fe}^{2+}$, the doublet (subpattern I) with the smaller splitting and the lower isomer shift to octahedral $\mathrm{Fe}^{3+}$. The presence of two $\mathrm{Fe}^{2+}$ patterns with roughly the same areas indicates that $\mathrm{Fe}^{2+}$ occupies two different cation positions in the ilvaite structure, e.g. $4 c$ and $8 d$ of space group Pbnm. Subpattern III is assigned to $\mathrm{Fe}^{2+}$ on the $4 c$ lattice site. This assignment is not based on any correlation between the Mössbauer parameters and the structural parameters of the site but rather on the temperature dependence of the Mössbauer parameters. The interactions between iron ions on the $4 c$ site and those on $8 d$ are expected to be weak since the sites are inequivalent and electron exchange between these two sites would be accompanied by appreciable activation and lattice deformation (elastic strain) energies. The Mössbauer parameters and their temperature dependence for the iron ions at the $4 c$ site should therefore be similar to those observed for isolated or noninteracting $\mathrm{Fe}^{2+}$ ions. This is indeed the case; subpattern III is unquestionably due to a $\mathrm{Fe}^{2+}$ ion throughout the investigated range of temperatures and pressures.

The behavior of subpattern II is in marked contrast to that of subpattern III. This subpattern can be only said to be "Fe ${ }^{2+}$-like," the degree to which it resembles a pattern expected for a well-defined $\mathrm{Fe}^{2+}$ electronic state being strongly pressure and temperature dependent. The relative intensity of this pattern is similar to that for $4 c$. However, the only other site occupied by iron to an appreciable extent is $8 d$ and therefore subpattern II arises from " $\mathrm{Fe}^{2+}$-like" ions occupying approximately one-half of the $8 d$ lattice sites.

Consequently, nominal $\mathrm{Fe}^{3+}$ has to fill the other half of the $8 d$ sites as first reported by Herzenberg and Riley [10] and subpattern I is assigned to a " $\mathrm{Fe}^{3+}$-like" ion on the $8 d$ lattice site.

The assignment of the subpatterns I-III seems to be confirmed, though the isomer shift of the $\mathrm{Fe}^{3+}$ doublet (subpattern I) even at $298 \mathrm{~K}$ is high for octahedral $\mathrm{Fe}^{3+}$ in either oxides or silicates [22]. It is still within the range of shifts observed for high spin ferric iron, e.g. $\delta=$ $0.49 \mathrm{~mm} \mathrm{~s}^{-1}$ for $\mathrm{Fe}^{3+}$ in $\mathrm{FeF}_{3}$, and the large isomer shift for $\mathrm{Fe}^{3+}(8 d)$ might simply be reflective of the low covalency of the $\mathrm{Fe}-\mathrm{O}$ bond at this site, which has $\mathrm{OH}^{-}$ as the source of one of the coordinated oxygens. The temperature dependence of the Mössbauer patterns does not, however, support this interpretation of the large isomer shift and the crystal/chemical iron species giving rise to subpattern I should be regarded as only " $\mathrm{Fe}^{3+}$. like."

Our results show that there is an additional broad adsorption between the high velocity components of the "Fe" ${ }^{3+}$-like" and "Fe ${ }^{2+}$-like" subpatterns, which was either not detected or considered unimportant in the earlier studies $[10,11,13]$. This absorption is very broad and cannot be described adequately by a single Lorentzian line. Consequently, we fitted it to two lines (subpattern IV) in order to simulate the line shape and no physical significance is ascribed to the magnitude of the quadrupole splitting. As shown in Tables 2 and 3 it is essential to an adequate fit of the spectra to include this broad absorption, whose relative intergrated intensity varies between $7.85 \%$ (No. 5) and $11.30 \%$ (No. 6). The interpretation of subpattern IV is not without some ambiguity. Similar features have been observed in mixedvalence ( $\mathrm{Zn}, \mathrm{Ti}, \mathrm{Fe}$ )-spinels and attributed to electron exchange between $\mathrm{Fe}^{2+}$ and $\mathrm{Fe}^{3+}$ on the octahedral sites [23]. An analogous interpretation seems to be appropriate for the $298 \mathrm{~K}$ spectra of ilvaite. At room temperature the extent of delocalization by this process is between 8 and 12\%. The low population of this delocalized electron state and the variation from sample to sample could be due to site inequivalence and variations in local symmetry occasioned by the presence of lattice defects and impurities, especially Mn. Even though the 
X-ray crystal structure indicates that $\mathrm{Fe}^{2+}$ and $\mathrm{Fe}^{3+}$ occupy the equivalent $8 d$ positions, the Mössbauer data indicate that the energy of the electron-delocalized state is unusually high for equivalent sites. For example, in $\mathrm{Eu}_{3} \mathrm{~S}_{4}[24]$ and $\mathrm{Fe}_{3} \mathrm{O}_{4}[1]$ the electron delocalization is complete at $300 \mathrm{~K}$.

\subsection{High temperature polycrystalline spectra}

The shape of the high temperature spectra is infuenced by several different effects which are readily associated with the different subpatterns. Subpattern III, which is assigned definitively to $\mathrm{Fe}^{2+}$ at the $4 c$ position, exhibits a temperature dependence of the isomer shift and quadrupole splitting associated with a unique lattice site and oxidation state of iron. The decrease in the isomer shift of approximately $4 \times 10^{-4} \mathrm{~mm} \mathrm{~s}^{-1}$ is in qualitative agreement with that expected for the Second Order Doppler shift; and the decrease in the electric quadrupole splitting is in accordance with the decrease that is usually observed for ferrous ions. Thus, the electronic structure of the $\mathrm{Fe}^{2+}$ ions at the $4 c$ site undergoes no qualitative changes with increasing temperature.

Even though it is clear that subpattern I still retains its "Fe $\mathrm{Fe}^{3+}$-like" character up to $330 \mathrm{~K}$, the temperature dependence of its isomer shift and quadrupole splitting in this temperature range is anomalous. The magnitudes of both of these quantities increase with increasing temperature contrary to the expected decrease. In principle, it is possible for the electric quadrupole splitting to increase with increasing temperature depending upon the temperature dependence of certain structural parameters but it is difficult to rationalize the increase in the isomer shift with temperature in the absence of qualitative changes in the electronic structure of the iron ion, i.e. of $\mathrm{Fe}^{3+}$ on $8 d$, giving rise to subpattern 1 . Consequently, we interpret the temperature dependence of the parameters of subpattern I as indicating a decrease in the electron density with temperature at the nucleus of the $8 d$ " $\mathrm{Fe}^{3+}$. like" ion. It has been noted in Section 4.1 that the isomer shift for this site is unusually large for $\mathrm{Fe}^{3+}$ in oxide and silicate structures and its anomalous temperature dependence may have the same origin as its unusually large magnitude at lower temperatures. The interpretation of the isomer shift of subpattern I at lower temperatures is still problematical since its value of $-0.57 \mathrm{~mm} \mathrm{~s}^{-1}$ still falls within the range of isomer shifts exhibited by orthodox $\mathrm{Fe}^{3+}$ ions; for example, a value of $0.49 \mathrm{~mm} \mathrm{~s}^{-1}$ is observed in $\mathrm{FeF}_{3}$ at $300 \mathrm{~K}$ [25]. Subpattern I cannot be assigned to a pure $\mathrm{Fe}^{3+}$ state at high temperatures and it loses its ferric-like character with increasing temperature. It is also interesting to note that the relative intensity of subpattern I does not change appreciably until $375 \mathrm{~K}$.

The temperature dependence of subpattern II between 300 and $375 \mathrm{~K}$ is also remarkable. In this case, the temperature dependence of the isomer shift and electric quadrupole splitting is in the expected direction, i.e. decreasing with increasing temperature, but the magnitude of the decrease in the isomer shift, $\sim-0.3$ $\mathrm{mm} \mathrm{s}^{-1}$, is at least a factor of 4 larger than the
$-0.07 \mathrm{~mm} \mathrm{~s}^{-1}$ expected for the SOD shift over this temperature interval. In this case, the variation in the parameters can be understood on the basis of an increase in the electron density at the nucleus of the $8 d \mathrm{Fe}^{2+}$ ion. As in the case of subpattern I, subpattern II also cannot be assigned to an orthodox $\mathrm{Fe}^{2+}$ state at higher temperatures. The small variations in the linewidths of subpattern II and the modest changes in intensities below $330 \mathrm{~K}$ are noteworthy.

Subpattern IV exhibits an appreciable temperature dependence below $330 \mathrm{~K}$ only in the magnitude of its apparent quadrupole splitting. In terms of the quadrupole doublet employed to fit this subpattern, this apparent decrease in quadrupole splitting is correctly considered as a decrease in the linewidth. This result suggests that the extent of the electron delocalization is not augmented appreciably by increasing temperature. The decrease in the width of subpattern IV implies a decrease in relaxation time for the electron transfer without a concomitant increase in the population of the delocalized electron state.

Above $375 \mathrm{~K}$ the high velocity components of subpatterns I, II and IV coalesce into a broad, structureless, absorption line, whose position is very similar to that of subpattern I, which has been assigned to a "Fe ${ }^{3+}$-like" species. Fitting four individual patterns to this spectrum is no longer justified. The spectrum has been fitted with two quadrupole doublets whose variation with temperature is in accordance with the expected SOD shift and the usual observations on the temperature dependence of the quadrupole splitting.

Except for two aspects, the interpretation of our high temperature results are in general agreement with those of some of the previous investigators $[11,13]$. Firstly, these investigators ignored the presence of subpattern IV in their data analysis and consequently, they considered the electron delocalization process as being evinced exclusively by subpatterns I and II. This is only true in part. Secondly, the high velocity components of subpatterns I and II are interpreted by these investigators as approaching each other at similar rates with increasing temperatures and coalescing at an intermediate velocity. It is clear from the present results that the high velocity components of subpatterns I, II and IV coalesce into a line whose position is much closer to that of the "Fe $\mathrm{Fe}^{3+}$. like," subpattern I component. Thus, in the high temperature limit, the pattern that results from the coalescence of subpatterns I, II and IV, which are undoubtedly due to $\mathrm{Fe}^{2+}$ and $\mathrm{Fe}^{3+}$ on the $8 d$ site, is assigned to species of iron ion that is more like an $\mathrm{Fe}^{3+}$ ion than either $\mathrm{Fe}^{2+}$ or an averaged $\mathrm{Fe}^{n+}$ species in which $\mathrm{Fe}^{3+}$ and $\mathrm{Fe}^{2+}$ are weighted equally. It is possible that the data of Gerard and Grandjean are similar to those presented here but the data are not reported in sufficient detail for a definitive comparison.

In a brief report of a recent ${ }^{37} \mathrm{Fe}$ Mössbauer investigation of ilvaite [15], the analysis and interpretation of the data were qualitatively different from any of those published thus far but since intensity data are not reported, the nature and origin of the differences cannot be assessed. 
There seems to be little doubt that the temperature dependence of the Mössbauer spectrum of ilvaite cannot be attributed to a simple mechanism of a rapid electron exchange between $\mathrm{Fe}^{2+}$ and $\mathrm{Fe}^{3+}$ ions at the $8 d$ sites. If this were the case, subpattern II would not exhibit the observed rapid variation in $e^{2} q Q / 2$ and $\delta$ with temperature; the intensity of subpattern IV would increase appreciably with temperature; and subpattern $V$ in the spectra above $375 \mathrm{~K}$ would have parameters intermediate to those of subpatterns I and II.

The high temperature spectra suggest the presence of two processes in ilvaite which influence its electronic structure; one process, evinced by subpattern IV, is associated with a rather limited electron delocalization between the iron ions on the $8 d$ sites. The other process involves primarily $\mathrm{Fe}^{2+}$ on the $8 d$ site and leads to reduction in the electron density at its nucleus and the development of a $\mathrm{Fe}^{3+}$-like electronic structure. In terms of the fraction of $8 d \mathrm{Fe}$ ions involved, this latter process is the more important of the two. The related changes in the $8 d \mathrm{Fe}^{3+}$ subpattern are not large in magnitude and seem to reflect nonlocalized changes in the electron density distribution on the $8 d$ sublattice, for example, an increase in the screening of the $\mathrm{Fe}^{3+}$ core as a result of an increase in the density of conduction electrons.

\subsection{High pressure spectra}

\subsubsection{High pressure spectra: Polycrystalline} samples. The primary effect of pressure on the spectrum of the polycrystalline sample is to shift the high velocity component of subpattern II to lower velocities. An analyses of the apparent quadrupole splitting and the isomer shift of this pattern (see Table 7) reveals a tendency for both the center of gravity and the quadruple splitting to decrease. This result is very similar to the effect of temperature (see Section 4.2) on subpattern II. The initially reported result of Gerard and Grandjean [11] that the primary influence of temperature is to reduce the apparent quadrupole splitting of subpattern II may also be extended to include the effects of pressure. It is still an open question at this stage whether the variation with temperature of the parameters of subpattern II is to be associated with a phase transition as suggested by Grandjean and Gerard [12]

The pressure dependence of the quadrupole splitting of subpattern I is similar to its temperature dependence which is again in contrast to the pressure dependence of the quadrupole splitting of all of the other patterns. The pressure dependence of the isomer shift is erratic and a definitive trend is not observed. Whether or not the isomer shift increases with pressure requires a more extensive set of measurements. Nonetheless, the effect of increasing pressure on the spectrum appears to be analogous to that of increasing temperature, see Fig. 12.

This is particularly true of subpattern IV as demonstrated by comparing Tables 5 and 7. If subpattern IV is, indeed, to be associated with electron exchange between different $\mathrm{Fe}$ sites, then the mechanism must be relatively insensitive to changes in temperature and pressure, e.g. the process is not thermally activated. These results suggest a tunnelling mechanism. The linewidth, relative

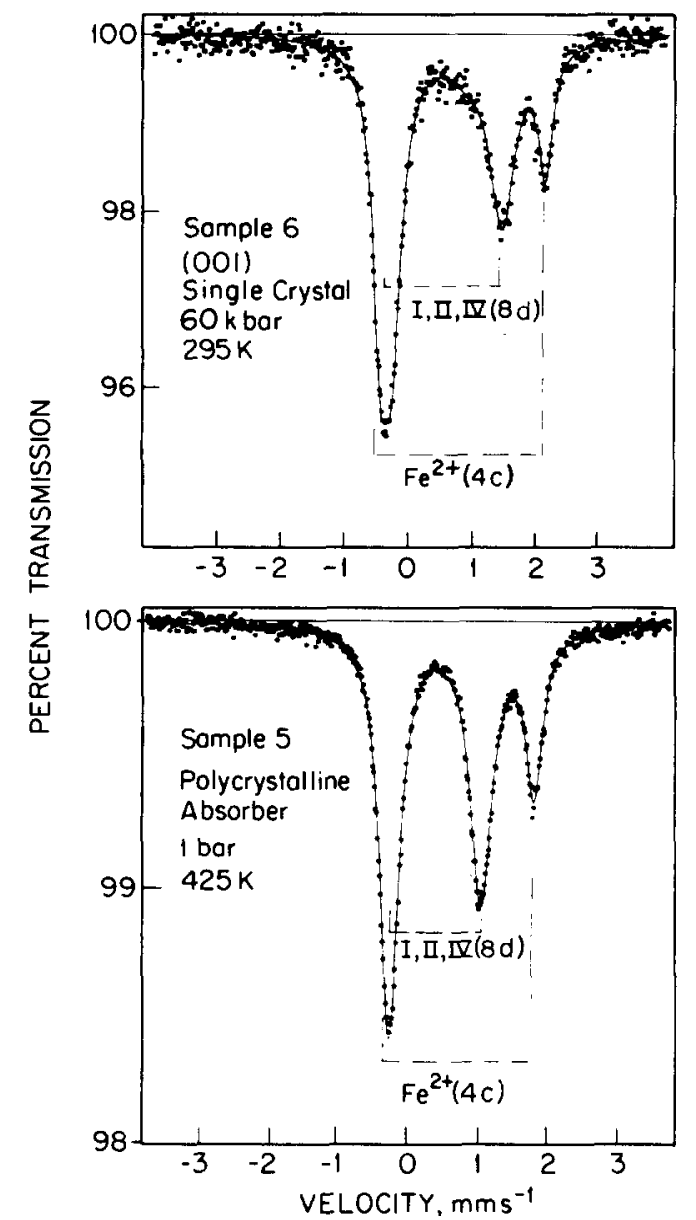

Fig. 11. Comparison of a high pressure, $298 \mathrm{~K}$ and a high temperature, 1 bar, spectrum of ilvaite, demonstrating the similarity of the effects of high pressure and high temperature on the ${ }^{57} \mathrm{Fe}$ Mössbauer spectrum.

intensity and their temperature dependence can be understood in terms of slightly differing local symmetries occasioned by substitutional impurities.

4.3.2 High pressure spectra: Single crystal samples. For the single crystal platelets the direction of propagation of the $\gamma$-rays and of the travel of the high pressure anvils is parallel to [100], [010] and [001] for the (100), (010) and (001) platelets, respectively (see Fig. 2). Thus, under the non-hydrostatic conditions that exist in the high pressure cell, the $a, b$ and $c$ axes experience the greatest compressions for the $(100),(010)$ and $(001)$ platelets, respectively. For the $(100)$ and $(010)$ platelets, the distance between the infinite chains of edge-sharing octahedra will, consequently, decrease with increasing pressure. The octahedra in different chains do not, however, share polyhedral elements and the chains are partially interleaved by $\mathrm{Si}_{2} \mathrm{O}_{7}$ groups. Thus, it is not expected that the electron delocalization in the $(100)$ and (010) platelets will undergo any dramatic changes for the modest pressures employed in this investigation.

For the (001) platelet, the direction of maximum compression, the $c$ axis, is parallel to the infinite chains and the intra-chain internuclear distance (shortest Fe-Fe distance is $2.83 \AA$ in the $c$-direction at 1 bar and $298 \mathrm{~K}$ ) 
between the iron ions is expected to decrease. In this case one expects the electron delocalization to be more sensitive to pressure, provided the compressibility along the $c$-axis is not too low. These expectations are borne out by the experimental results. The results for the (001) single crystal platelet also verifies that subpattern II is shifted to lower velocities with increasing pressure. The significant feature of these results is the qualitatively greater effect of pressure on the spectrum for the $(001)$ platelet which indicates that the electron delocalization process takes place preferentially in the infinite chains. Thirty kilobar pressure has an influence on the spectrum of an (001) platelet that requires at least 60 kilobars for polycrystalline, $(100)$ and $(010)$ samples. The results on the $(100)$ and $(010)$ platelets are, therefore interpretable in a manner similar to that employed for the high pressure spectra of polycrystalline absorbers.

It is significant that the $60 \mathrm{~kb}$ spectrum of the $(001)$ platelet is similar to that of the polycrystalline sample at about $373 \mathrm{~K}$. It is also remarkable that the $\mathrm{Fe}^{2+}$ spectrum becomes more "Fe ${ }^{3+}$-like" with either increasing pressure or temperature. Indeed, the "Fe ${ }^{2+}$-like" spectrum is indistinguishable from the " $\mathrm{Fe}^{3+}$-like" spectrum at $373 \mathrm{~K}$ and 1 bar pressure and at $298 \mathrm{~K}$ and $30 \mathrm{~kb}$ for the $(001)$ single crystal specimen. The coalescence of subpatterns I and II does not produce a pattern intermediate between the individual $\mathrm{Fe}^{3+}$ and $\mathrm{Fe}^{2+}$ lines as is usually the case for electron exchange [24]. Thus the more important effect of pressure (and temperature) is not to lead to a transfer of electron density from $\mathrm{Fe}^{2+}$ to $\mathrm{Fe}^{3+}$ within the chains but rather to a transfer of electron density from the $\mathrm{Fe}^{2+}$ ions into a band-like state, with perhaps a general redistribution of electron density by means of screening processes.

\section{CONCLUSIONS}

Four partially resolved patterns are required to account for the spectra of ilvaites for temperatures below $340 \mathrm{~K}$ and for pressures up to $60 \mathrm{kbar}$ for polycrystalline samples at $298 \mathrm{~K}$.

The four patterns are assigned to the following crystal/chemical states of iron: (a) Subpattern $I$ is due to a "Fe $\mathrm{F}^{3+}$-like" ion on the $8 d$ lattice site for which the electron density at the nucleus decreases modestly with increasing pressure and temperature. (b) Subpattern II arises from a " $\mathrm{Fe}^{2+}$-like" ion on the $8 d$ lattice site which with increasing temperature and pressure becomes progressively more ferric-like. (c) One pattern, subpattern III is due to a well-defined $\mathrm{Fe}^{2+}$ ion on the $4 c$ site throughout the temperature and pressure range investigated. (d) Subpattern IV is attributed to a component of a relaxation spectrum due to electron exchange between $\mathrm{Fe}^{2+}$ and $\mathrm{Fe}^{3+}$ ions on the $8 d$ lattice sites.

The behavior of the ${ }^{57} \mathrm{Fe}$ Mössbauer spectra of ilvaites which is critical to an understanding of its electronic structure and possible interactions between the $8 d \mathrm{Fe}^{2+}$ and $\mathrm{Fe}^{3+}$ ions are the following: The first is the dramatic decrease with increasing pressure or temperature of the apparent quadrupole splitting and isomer shift of the "Fe $\mathrm{F}^{2+}$-like" subpattern II and the less dramatic but qual- itatively more anomalous increase of the quadrupole splitting and isomer shift for the "Fe ${ }^{3+}$-like" subpattern I. These coupled changes in the spectrum lead to a coalescence of these two patterns at temperatures above $350 \mathrm{~K}$ at 1 bar pressure and at $298 \mathrm{~K}$ and pressures above $28 \mathrm{kbar}$ and $60 \mathrm{kbar}$, respectively, for a single crystal (001) platelet and either (100), (010) platelets or polycrystalline samples. Secondly, there is the weak temperature dependence of the parameters of subpattern IV which is attributed to electron exchange between iron ions on the $8 d$ lattice sites in a manner analogous to that proposed for certain paramagnetic spinel ferrites. We therefore propose that the electron delocalization in ilvaite be understood in terms of two distinguishable but connate processes. One is electron exchange between $\mathrm{Fe}^{2+}$ and $\mathrm{Fe}^{3+}$ ions resulting in a mixing of $\mathrm{Fe}^{2+}$ and $\mathrm{Fe}^{3+}$ states to the extent of only $5-15 \%$. The other electron delocalization process involves primarily $\mathrm{Fe}^{2+} 8 d$ ions in such a way that they become more $\mathrm{Fe}^{3+}$ like with increasing pressure and temperature, that is to say a process such as the following

$$
\mathrm{Fe}^{2+} \rightarrow \mathrm{Fe}^{3+}+e^{--}
$$

In this process the $e^{-}$is intrinsically delocalized and does not occupy another localized state. There are, to be sure, some interactions between the delocalized electrons and the $\mathrm{Fe}^{3+}$ ions in the chains but this interaction appears to be one simply of screening.

The more important process leading to a delocalization of the electrons is associated with the above-mentioned anomalies in the shifts of patterns I and II. As noted, pattern II showed the greatest changes and it is reasonable to conclude that delocalization is taking place primarily at the $\mathrm{Fe}^{2+} 8 d$ ions. The population of the conduction band by the $3 d$ electrons of the $\mathrm{Fe}^{2+} 8 d$ ion increases with increasing temperature by means of thermal activation of electrons into the conduction band or through a temperature dependent band width. Either one or both of these mechanisms for the increase in the density of conduction electrons is supported by our high temperature and high pressure measurements. The width of the conduction band in a material such as ilvaite is expected to be relatively narrow and the changes observed in the spectrum of the subpattern for the $8 d \mathrm{Fe}^{3+}$ ion are due to the increase in the screening of the $\mathrm{Fe}^{3+}$ core as the density of the conduction electrons increases. In this case the electronic state of the $\mathrm{Fe}$ ions does not reflect an equal weighting between $\mathrm{Fe}^{2+}$ and $\mathrm{Fe}^{3+}$, but rather is nearer that of an $\mathrm{Fe}^{3+}$ ion since the buildup of charge at the $8 d$ sites due to screening is not expected to amount to 0.5 electrons.

The extent of the electron delocalization arising from electron exchange between the $8 d \mathrm{Fe}^{2+}$ and $\mathrm{Fe}^{3+}$, (e.g. the intensity of subpattern IV) ions is different in samples having different origins and composition. The question of the influence of composition on electron delocalization in natural ilvaites must be regarded as still unresolved.

In addition to investigations of ilvaites with systematically controlled compositions, a rigorous fit of a model 
of dynamic electron exchange to the ${ }^{57} \mathrm{Fe}$ Mössbauer spectrum is desirable. Such a fit, however, requires a detailed knowledge of the sign and orientation of the electric field gradient at the various sites and must await more extensive measurements on single crystals, which are currently underway. The suggestion of Gerard and Grandjean regarding the possibility of a phase transition at about $340 \mathrm{~K}$ is also deserving of further investigation.

It is clear, however, that despite the need for further study, the ${ }^{57} \mathrm{Fe}$ Mössbauer spectra of ilvaite exhibit the qualitative characteristics expected when there are strong interactions between $\mathrm{Fe}^{2+}$ and $\mathrm{Fe}^{3+}$ ions on equivalent lattice sites. The electron exchange process is not explicable in terms of a single mechanism but the extent to which this is an intrinsic characteristic of pure $\mathrm{CaFe}_{3} \mathrm{Si}_{2} \mathrm{O}_{7} \mathrm{O}(\mathrm{OH})$ must await further investigations of high purity samples.

Acknowledgements-The authors are grateful for the provision of facilities during part of this investigation by S. S. Hafner. Technical assistance was cheerfully provided by K. Schaumann, R. Vogel, Lu San Pan and K. Runge. It is a pleasure for B. J. Evans to acknowledge the award of a fellowship by the Alexander von Humboldt Foundation and partial support of this investigation by the National Science Foundation.

\section{REFERENCES}

1. Evans B. J., Experimental studies of the electrical consuctivity and phase transition in $\mathrm{Fe}_{3} \mathrm{O}_{4}$, In AIP Conf. Proc. (Edited by C. D. Graham, Jr., G. H. Lander and J. J. Rhyne), Vol. 24, pp. 73-78. New York (1975).

2. Leung L. K., Morrish A. H. and Evans B. J., Phys. Rev. 13, 4069 (1976).

3. Allen G. C. and Hush N. S., Intervalence absorption, Part 1, In Prog. Inorg. Chem. (Edited by F. A. Cotton), Vol. 8, pp. 357-390. Wiley, New York (1967).
4. Robin M. B. and Day P., Adv. Inorg. Radiochem. 10, 247 (1967).

5. Smith C. and Strens R. G. J., Intervalence transfer absorption in some silicate, oxide and phosphate minerals, In The Physics and Chemistry of Minerals and Rocks (Edited by R. G. J. Strens), pp. 583-612. Wiley, New York (1976).

6. Belov N. V. and Mokeeva V. I., Trude. Inst. Krist. Akad. Nauk. SSSR 9, 49 (1954).

7. Beran A. and Bittner H., Tschermaks Min. Petr. Mitt. 21, 11 (1979).

8. Haga N. and Takeuchi Y., Zeit. Kristallogr. 144, 161 (1976).

9. Bancroft G. M., J. de Phys. C-2 C2-464 (1979).

10. Herzenberg C. L. and Riley D. L., Acta Cryst. A25, 389 (1969).

11. Gerard A. and Grandjean F., Solid State Commun. 9, 1845 (1971).

12. Grandjean F. and Gerard A., Solid State Commun. 16, 553 (1975).

13. Heilmann I. U., Olsen N. B., Olsen J. S., Phys. Script. 15, 285 (1977).

14. Amthauer G. and Evans B. J., Phys. Chem. Minerals 3, 55 (1978).

15. Nolet D. A. and Burns R. G., Geophys. Res. Lett. 4, 821 (1978).

16. Ruby S. L., Why MISFIT when you already have $\chi^{2}$ ?, In Mössbauer Effect Methodology (Edited by I. Gruverman), Vol. 8, pp. 263-276. Plenum Press, New York (1973).

17. Decker D. L., Bassett W. A., Hall H. T. and Barnett J. D., J. Phys. Chem. Ref. Data 1, 773 (1972).

18. Cannon J. F., J. Phys. Chem. Ref. Data 3, 781 (1974).

19. Amthauer G., Annersten H. and Hafner S. S., Zeit. Kristal$\log r 143,14$ (1976).

20. Ok H. N. and Evans B. J., Phys. Rev. 14, 2956 (1976).

21. Schilling J. S., Klein U. F. G. and Holzapfel W., Rev. Sci. Instrum. 45, 1353 (1974).

22. Greenwood N. N. and Gibb T. C., Mössbauer Spectroscopy, p. 150. Chapman \& Hall, London (1971).

23. Lotgering F. K. and van Diepen A. M., J. Phys. Chem. Solids 38, 565 (1977).

24. Berkooz O., Malamud M. and Shtrikman S., Solid State Commun. 6, 185 (1968).

25. Wertheim G. K., Guggenheim H. J. and Buchanan D. N. E., Phys. Rev. 169, 465 (1968). 